https://doi.org/10.48009/1_iis_2005_21-27

\title{
NORMALIZATION SHOOTOUT: A COMPETITIVE GAME THAT IMPACTS STUDENT LEARNING
}

\author{
Ronnie A. Fanguy, Nicholls State University, Ronnie.Fanguy@ Nicholls.edu \\ Betty A. Kleen, Nicholls State University, Betty.Kleen@ Nicholls.edu
}

\begin{abstract}
The normalization shootout described in this paper is an effective way to challenge students to learn normalization concepts. Students compete two at a time in a tournament-style series of matches. Tournament questions require students to identify closures, the key, and highest normal form for relations and their corresponding functional dependencies. Since introducing a shootout game in the fall of 2004, initial test scores have improved over previous semesters, retesting indicates those who advance in the tournament retain more at the end of the semester, and written feedback indicates students perceive the game is an effective teaching tool.
\end{abstract}

Keywords: Educational games, active learning, competition, motivation

\section{INTRODUCTION}

Many of us can think back and remember days when a teacher would make learning fun by involving the class in some type of game to help reinforce knowledge and/or concepts. Throughout our elementary and secondary education years, we may have participated in competitions such as spelling bees, geography bees, or quiz bowls. The normalization shootout described in this paper is an adaptation of such exercises. The "shootout" is offered as an additional way to challenge students to learn database normalization concepts.

\section{GAMES AS EFFECTIVE TEACHING TOOLS}

Does the "fun" aspect of learning have to be removed once the learner leaves preschool and elementary school behind? Hundreds of articles can be found by searching a combination of key words "learning," "teaching," and "games." In his 1999 article, Ruben offered a 30+ year overview of experiential instruction theory and practice (6). He reported how various activelearning instructional methods such as games have had an impact on teaching concepts since the 1960 's. Various levels of education beyond elementary school now recognize the value of games as a tool for teaching facts and concepts.

Wheatley wrote about using numerous experiential exercises derived from children's games for management education (8). Gremmen and Potters reported using games to enhance learning effectiveness in economic education (3). Cook and Hazelwood applied a competitive game show activity to increase student advance preparation and class participation in accounting (2). Specifically within the computer science and information systems fields, Becker reported on an effective way to use minesweeper and asteroids as programming applications (1). Siegfried used "Chomp," an educational strategy game, in high school programming courses (7). Lawrence's students were motivated to learn advanced programming when he used a competitive game to teach server architecture (4). The game also improved students' opinions of the course. 
A common observation that emerged from the above articles is that student learning did occur, and the instructors helped make learning fun for the students. This was true no matter what the discipline and no matter what the age of the learners. The "normalization shootout" described in the next section is yet another game that can be used effectively in teaching database design at any educational level.

\section{TEACHING NORMALIZATION}

The authors of this paper use a normalization shootout in the database design and implementation course in the computer information systems curriculum. The lesson on normalization begins with an examination of the three fundamental concepts on which the normal forms up to third normal form (3NF) are built-functional dependencies, closures, and keys. A functional dependency is simply a constraint between two sets of attributes in a relation, such that every tuple that has identical values for the first set of attributes must have identical values for the attributes in the second set of attributes. This is the most basic foundation for the remainder of the normalization concepts covered. Secondly, we move forward with the question "What are all the attributes functionally dependent on a given set?" This is the precise question we must answer if we wish to find the closure. The third fundamental concept covered is that of a key. A set of attributes $\mathrm{k}$ within a relation is a key of the relation if it satisfies two properties: (1) all the attributes in the relation are functionally dependent on $\mathrm{k}$ and (2) no subset of $\mathrm{k}$ also has property 1.

After examining these fundamental concepts, we introduce the normal forms-first normal form (1NF), second normal form (2NF), and third normal form (3NF) — as a sequence of steps that leads us closer and closer to a database structure that is well designed. In normalizing to first normal form (1NF), we ensure that no relation within the database contains any repeating groups - or any combination of multivalued, composite attributes. In $2 \mathrm{NF}$, we examine the database's relations to identify whether any nonkey attributes are partially dependent upon the keys of the relations. If any are found, the relations containing them are decomposed so that the partial dependency no longer exists-while preserving the relevant functional dependencies. Finally, we examine the relations composing the database to identify whether any nonkey attributes are transitively dependent upon the keys of the relations. If any are, then another decomposition step is performed to remove these attributes from their original relations and regroup them in such a way as to ensure that the relevant functional dependencies still hold. From a technical perspective, this is actually the definition of Boyce-Codd normal form rather than third normal form; however, we follow the conventions of authors such as Pratt and Adamski in not distinguishing between them except for a brief mention of the distinction within class (5).

\section{THE GAME: THE "NORMALIZATION SHOOTOUT”}

The game we employ to encourage students to study and understand normalization is dubbed a "normalization shootout." The game involves students facing off two at a time to compete in a tournament-style series of matches to earn bonus points by correctly answering questions focused on the normalization topics that we have discussed. Different types of targets and scoring implements such as suction balls, nerf balls, or sponge balls could be used for the game. When the game is played using a basketball theme, answer options are set up behind toy 
basketball hoops. To win a match, a student must correctly answer 3 out of 5 multiple-choice questions faster than his/her opponent. However, simply knowing the correct answer is not enough. The student must "shoot the hoop" representing the correct answer before the opponent in order to earn the point for the current question. Competitors are positioned near targets to help ensure that poor aim does not contribute to inability to "score."

The multiple-choice normalization questions for the entire tournament are organized into a Powerpoint presentation, in a very active format. Instead of simply displaying the question on a pull-down screen, the presentation appears on a touch-activated Smartboard, which allows control of the computer by touching the board itself. The authors' original plan was to have students aim at the Smartboard. Some type of affirmative sound-such as cheering or bells ringing - would occur when students scored the correct answer and a negative sound — such as a buzzer or explosion-would occur when the wrong answer was activated. However, such activity could possibly damage the touch sensitivity of the Smartboard, so an alternative plan emerged. Instead, we set up targets (small basketball backboards and hoops) representing the answers to the multiple-choice questions; contestants then shoot the hoop of the correct answer choice after reading the questions displayed on the Smartboard.

To encourage students to participate and put forth their best effort, students are awarded bonus points. Each student who participates earns at least one bonus point. Students winning each round earn additional bonus points and advance to the next round of competition. With each new round of matches, the questions become progressively more challenging. Based on class size and time allotted to the game, our tournament is organized into three rounds of matches.

\section{First Round}

In the first round, students are shown a relation and a set of functional dependencies that hold over these attributes. In this round, the relation and set of functional dependencies does not change during the match. For each match in the first round, students must identify the following:

Questions 1-3: $\quad$ closures

Question 4: the key

Question 5: $\quad$ the highest normal form

Since closure is used to define a key and keys are used to define the normal forms, this sequence is progressively challenging. Play stops after one contestant earns three points; therefore, the more challenging questions are only posed if contestants are equally prepared for competition.

\section{Second Round}

In the second round, the level of difficulty increases. In this round, the relation is static; however, the set of functional dependencies that hold over the relation changes after the first question. The sequence of questions adheres to the following pattern.

Question 1: $\quad$ identification of the highest normal form

Questions 2 \& 3: $\quad$ as the set of functional dependencies changes, the key and highest normal form must be identified 
Question 4: $\quad$ identification of either a partial dependency or a transitive

Question 5: $\quad$ identification of a valid decomposition.

\section{Additional Rounds}

Additional rounds may be added as needed to restrict the number of students. However, when there are only a few students remaining - typically around 3-these students are dubbed the champions of the normalization shootout and are given an opportunity to earn additional bonus points in the challenge round. As soon as a contestant wins a match that advances him/her to the final round, the contestant is given a set of three more challenging normalization questions to answer while play continues for the remaining matches. Once the challenge round begins, the remaining contestants individually present their answers to the class (while the other challenge round contestants remain sequestered in a nearby room). Correctly explaining how the answers were determined earns the student additional bonus points.

\section{Reducing Random Guessing}

One lesson we learned after our inaugural game is that some mechanism must be in place to discourage or prevent random guessing. The first time the game was played some students were simply guessing at the answers. If left unabated, this problem enables the person taking the most shots most quickly to almost always triumph over the careful thinker-especially for problems that require some thought. This goes against the primary goal of reinforcing the students' understanding of the concepts. There are many ways that guessing can be discouraged. We choose to allow contestants to shoot only one hoop before the other contestant takes a shot. In this way, only after both contestants get an initial guess, may another choice be made. We believe that this will discourage random guessing because if an opponent randomly guesses his/her answer, it will:

1. likely be wrong ( 4 out of 5 incorrect answers for most questions and 2 out of 3 for a few).

2. increase the probability that the guesser's opponent will be able to randomly guess correctly, as one fewer wrong answer will be available

3. relieve the pressure off of the opponent to answer quickly.

The only exception we allow is if a contestant takes too long in answering. Care must be taken to ensure that the opponent does not simply waste time.

\section{IS THE GAME AN EFFECTIVE USE OF CLASS TIME?}

The authors have used the game during the Fall 2004 and Spring 2005 semesters. Based on two semesters' analysis, we believe it is a fun activity that has contributed to student learning. Several benefits have been identified.

\section{Students Enjoy the Game}

Based on verbal and nonverbal feedback during the game, most students find the game quite fun and have a good time as play begins and progresses. We believe that the game engages students in active learning. The standard alternative is to go over homework problems that were assigned 
but only completed by a few conscientious students. During a standard homework review, many students will only half-heartedly attempt to understand-relying on their written notes to somehow make sense when they finally decide to look at them again the night before the test. Instead, during the shootout the students are challenged to recall and apply concepts in an interactive game environment. We believe that making students aware that bonus points are available to those who do well in the game will increase the likelihood that they will study their notes and review their homework assignments before test time approaches. We also hope to increase the student's attentiveness and desire to understand the material.

At the end of each match, we spend a few moments reviewing the questions to make sure that the students participating understand the answers. Although it prolongs the game time, we believe it is important to take advantage of the situation. Both during and after the match, the interest is heightened for many of the students in the room. For obvious reasons, the current contestants are engaged in figuring out the answers, and they are particularly interested in the explanations of the questions. However, in addition to the contestants, the spectators for the current match tend to be actively involved in understanding the problems during and after the match-as many want to see if they can figure out the answers before their classmates who are competing. While this is true for many, it is especially true of those who are awaiting subsequent matches - as a mental warm up and as a way of sizing up the competition.

\section{Students Learn from Other Students}

In the challenge round, students must explain their answers to rather difficult problems. This gives an opportunity for the class to learn from the students who understand the concepts the best (as is evidenced by their making it to the challenge round). As instructors, we sometimes have a hard time seeing the material from the student's perspective. Something that seems obvious to someone who has a firm grasp of the material is often not obvious to those being introduced to it for the first time. Therefore, the challenge round gives an opportunity for light to be shed on normalization from a student's point of view.

\section{Student Understanding of Materials Has Increased}

The shootout game is used as a reinforcement tool during instruction of normalization. Students are tested on normalization soon after. Table 1 contains details of student scores on the test during the past four terms. The game was used during Fall 2004 and Spring 2005. The average score for students who played the normalization shootout was higher than for those who did not.

To measure how well students retained what they had learned, the same test was administered at the very end of the semester. For all five test topics, those who won at least one match of the game retained the knowledge of how to do the normalization better than the group of students who either lost their first and only match or did not participate (see Table 2).

\section{Students Perceive the Game Has Value}

The authors also surveyed the Spring 2005 students to obtain more concrete feedback in six areas concerning their perceptions of the usefulness of the shootout. As Figure 1 illustrates, students 
strongly agreed they enjoyed the game. They also agreed they studied for the game, would study for the game if it were to be played again, found the game helped them understand normalization concepts, and felt it was an effective use of class time.

Table 1. Comparison of Database Design Test Scores for Students by Level of Participation in Normalization Shootout Game

\begin{tabular}{cccc} 
Semester & Number & Average & Std \\
\hline Students Who Participated in the Normalization & Shootout Game & \\
\hline 2005 Spring & 14 & 84.1 & 7.1 \\
2004 Fall & 9 & 76.7 & 11.8 \\
Summary & 23 & 81.2 & 8.9
\end{tabular}

Students Who Did NOT Participate in the Normalization Shootout Game

$\begin{array}{cccc}\text { 2005 Spring } & 0 & \text { N/A } & \text { N/A } \\ \text { 2004 Fall } & 5 & 69.3 & 12.9 \\ \text { 2004 Spring } & 14 & 74.5 & 12.6 \\ 2003 \text { Fall } & 18 & 67.7 & 13.0 \\ \text { Summary } & 37 & 70.5 & 12.9\end{array}$

Table 2. Percentage Difference in Retake Scores for Normalization Examination for Students Enrolled Fall 2004 and Spring 2005

\begin{tabular}{lcc}
\multicolumn{1}{c}{ Topic } & $\begin{array}{c}\text { Students Who Did NOT } \\
\text { Advance or Did not Participate }\end{array}$ & $\begin{array}{c}\text { Students who Advanced } \\
\text { in the Game }\end{array}$ \\
\hline Functional Dependencies & $-8.8 \%$ & $-1.7 \%$ \\
Definitions & $-3.9 \%$ & $0.5 \%$ \\
Highest Normal Form & $-5.0 \%$ & $4.9 \%$ \\
Identify the Key & $0.0 \%$ & $3.1 \%$ \\
Decomposition & $-8.6 \%$ & $6.1 \%$ \\
Total Score & $-4.9 \%$ & $2.2 \%$
\end{tabular}

\section{CONCLUSIONS AND IMPLICATIONS}

Games can be valuable tools to enhance student learning. The "normalization shootout" game described in this paper is an effective way to challenge students to learn normalization concepts. Data show it initially enhances student understanding of the material, contributes to retention of that knowledge, and helps students learn from each other. Students surveyed also perceive the game has value. The authors plan to continue to use the game and gather additional semesters' data concerning its effectiveness. Other information systems educators may also wish to explore the use of gaming as a teaching tool to increase student learning. 


\section{Average Responses \pm Standard Deviation}

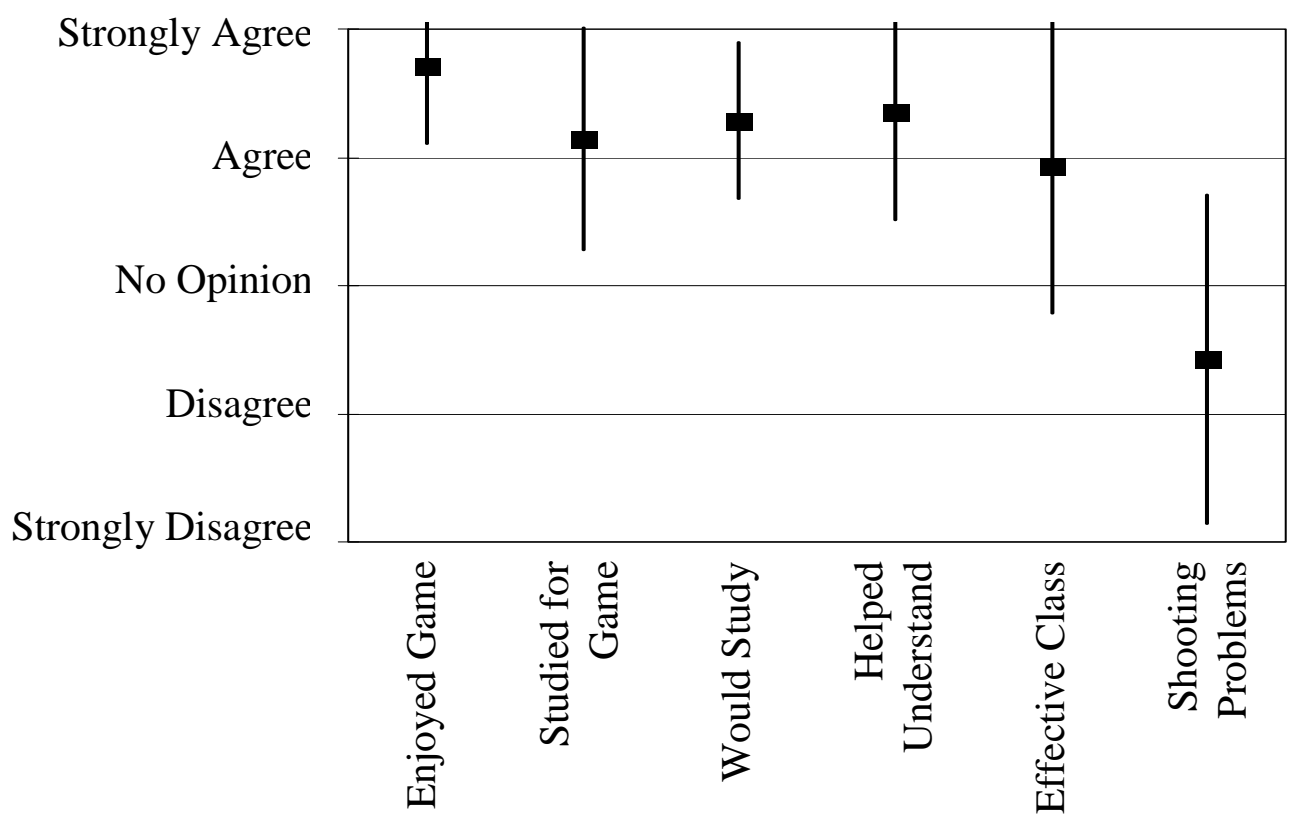

Figure 1. Students' Opinions Regarding Game

\section{REFERENCES}

1. Becker, L. (2001). Teaching with games: The minesweeper and asteroids experience, Journal of Computing in Small Colleges, 17(2), 23-33.

2. Cook, E, \& Hazelwood, A. (2002). An active learning strategy for the classroom - "Who wants to win ... some mini chips ahoy?" Journal of Accounting Education, 20(4), 297-307.

3. Gremmen, H., \& Potters, J. (1997). Assessing the efficacy of gaming in economic education. Journal of Economic Education, 28(4), 291-303.

4. Lawrence, R. (2004). Teaching data structures using competitive games, IEEE Transactions on Education online.

5. Pratt, P. \& Adamski, J. (2002). Concepts of Database Management. Boston: Course Technology.

6. Ruben, B. (1999). Simulations, games, and experience-based learning: The quest for a new paradigm for teaching and learning. Simulation \& Gaming, 39(4), 498-505.

7. Siegfried, E. (April, 2002). One of my favorite assignments: "Chomping" into computer science, Journal of Computer Science Education online. ERIC No. EJ656050.

8. Wheatley, W. (1999). Enhancing the effectiveness and excitement of management education: A collection of experiential exercises derived from children's games. Simulation \& Gaming, 39(2), 181-198. 